\title{
Aspectos metodológicos na construção de projetos de pesquisa em Nutrição Clínica
}

\author{
Methodological aspects in the \\ development of research projects \\ in Clinical Nutrition
}

Deyliane Aparecida de Almeida PEREIRA ${ }^{1}$

Sarah Aparecida VIEIRA ${ }^{2}$

Aline Siqueira FOGAL ${ }^{3}$

Andréia Queiroz RIBEIRO ${ }^{4}$

Sylvia do Carmo Castro FRANCESCHINI ${ }^{4}$

RE S U M O

O objetivo deste texto é fomentar a reflexão e a crítica no processo de elaboração de projetos de pesquisa em nutrição clínica. São apresentados aspectos relativos a evidências, validade e confiabilidade dos resultados dos estudos nesse campo. É imprescindível que ocorra adequado planejamento da pesquisa, desde a definição do delineamento e do tipo do experimento, passando pelos aspectos éticos, escolha da população e cálculo do tamanho amostral até a avaliação da factibilidade dos riscos envolvidos na sua condução. Após a coleta das informações, as etapas seguintes correspondem à descrição dos resultados, análise estatística, verificação da consistência desses resultados e, ao final, sua adequada interpretação.

Termos de indexação: Amostra. Análise estatística. Pesquisa.

A B S T R A C T

This text aims to foster the reflection and criticism in the process of developing research projects in clinical nutrition. We present aspects regarding the evidence, validity, and reliability of results of studies in this field.

1 Faculdades Sudamérica, Curso Educação Física. Cataguases, MG, Brasil.

2 Universidade Federal de Viçosa, Departamento de Nutrição e Saúde, Programa de Pós-Graduação em Ciência da Nutrição.

Viçosa, MG, Brasil.

3 Nutricionista. Viçosa, MG, Brasil.

${ }^{4}$ Universidade Federal de Viçosa, Departamento de Nutrição e Saúde. Av. PH Rolfs, s/n., 36570-900, Viçosa, MG, Brasil. Correspondência para/Correspondence to: SCC FRANCESCHINI. E-mail: <sylvia@ufv.br>. 
Appropriate study planning is critical, from defining the design and type of experiment, going through the ethical aspects, population choice, and calculation of sample size, to the assessment of the feasibility of the risks involved in study execution. Once the information is collected, the next stages correspond to the description of the results, statistical analyses, verification of the consistency of these results, and ultimately their correct interpretation.

Indexing terms: Sample. Statistical analysis. Research.

\section{N T R O D U ÇÃ O}

Este texto foi elaborado com o objetivo de fomentar a reflexão e a crítica no processo de elaboração de projetos de pesquisa em nutrição clínica.

Atualmente a Prática Baseada em Evidências (PBE) é um método de pesquisa utilizado nas diversas áreas de conhecimento, especialmente em Saúde, objetivando integrar resultados/achados de estudos para orientar a tomada de decisão. Essa estratégia procura compreender e empregar o método científico, para que se avaliem e se sistematizem os resultados, de forma a contribuir para a tomada de decisões ${ }^{1}$.

A Prática Baseada em Evidências divide-se em etapas, iniciadas com a identificação do problema clínico que influenciará a formulação do problema de pesquisa. Em seguida, objetivando sanar esse problema, são buscadas na literatura evidências relativas a procedimentos terapêuticos, utilizando estratégias de revisões sistemáticas e a sua avaliação em face do problema clínico formulado'. Cruz \& Pimenta² sugerem alguns questionamentos para avaliação das evidências encontradas, a saber: 1) "Os resultados são válidos? Quais são os resultados? Os resultados me ajudarão no cuidado do paciente?" (p.417). Após a exploração e avaliação do material obtido quanto à acurácia, validade e relevância, avalia-se a sua aplicabilidade na clínica e, em caso de resultado positivo, implementam-se as terapêuticas no cuidado do paciente, de acordo com as evidências disponíveis na literatura. Por fim, após realização do tratamento, avaliam-se os resultados da mudança'.

Nesse sentido, nota-se que, na prática clínica, os princípios da PBE são indispensáveis para que haja diagnóstico e intervenção efetiva, especialmente na área da Nutrição. Assim, é essencial que se aprecie a validade de um teste de diagnóstico (delineamento dos estudos), a capacidade do teste em fornecer respostas específicas ao problema de investigação, o desempenho do teste em avaliar a magnitude dos achados, e a adequabilidade do teste no contexto clínico diagnosticado ${ }^{2}$

Uma das estratégias de avaliação das evidências científicas é a classificação dos estudos quanto à sua força (Quadro 1) ${ }^{3}$. O nível indica quão forte e bem delineado é o estudo, ou seja, qual a credibilidade científica para a tomada de decisão. Contudo, quando não há pesquisas com nível alto de evidência, analisa-se qual é aquela com melhor classificação disponível, e não a melhor possível.

Destarte, a evidência científica é delimitada pela validade e confiabilidade da pesquisa, a fim de evitar conclusões/inferências espúrias². A validade identifica se o estudo mede aquilo a que realmente se propõe, ou seja, se os resultados são verdadeiros ou quanto se afastam da verdade $^{4}$. Para isso, o pesquisador precisa determinar os indicadores de sensibilidade (detectar o seu objeto de estudo) e especificidade (detectar somente o seu objeto de estudo), bem como seus valores preditivos positivos (probabilidade de o achado ser realmente seu objeto de estudo) e negativos (probabilidade de o achado não ser realmente seu objeto de estudo) $)^{5}$. Já a confiabilidade se refere à concordância ou consistência dos resultados e à ausência de erro aleatório, ou seja, o estudo deve ser reprodutível em situações similares e possuir regularidade no procedimento e clareza 4 .

No processo de análise e avaliação das informações em nutrição clínica é indispensável 
Quadro 1. Análise das evidências nas pesquisas.

\begin{tabular}{ll}
\hline Nível de evidência & Fontes de evidência \\
\hline I & Metanálise de múltiplos estudos controlados. \\
II & Estudo experimental individual. \\
III & Estudo experimental com grupo único, não randomizado, controlado, com pré- e pós-teste, ou estudos tipo \\
& caso-controle. \\
IV & Estudo não experimental, como pesquisa descritiva correlacional, pesquisa qualitativa ou estudo de caso \\
V & Relatório de casos ou dados obtidos sistematicamente, de qualidade verificável, ou dados de programas de \\
& avaliação. \\
VI & $\begin{array}{l}\text { Opinião de autoridades respeitadas (como ou opinião de um comitê de peritos, incluindo suas interpretações não baseadas em pesquisa. Esse nível } \\
\text { também inclui opiniões de órgãos de comunicação ou legais. }\end{array}$ \\
\hline
\end{tabular}

Fonte: Stetler et al. ${ }^{3}$

a crítica do material obtido, desde os resultados até os procedimentos metodológicos empregados. Ademais, é necessário proceder a cinco questionamentos: avaliação da relevância da pesquisa, avaliação do delineamento do estudo, avaliação dos resultados obtidos para solução do problema, avaliação da consistência e confiabilidade dos resultados, e avaliação quanto à contribuição dos dados para a tomada de decisão.

A avaliação da relevância da pesquisa deve se ater à importância da temática para a comunidade científica e a tomada de decisão na prática profissional, bem como à novidade e relevância do objeto de estudo ${ }^{1,2}$.

No que concerne ao delineamento, o pesquisador deve se fundamentar em objetivos claros, concisos e, principalmente, tangíveis. O delineamento do estudo se refere ao desenho da pesquisa, onde se avaliam os resultados em termos de consistência, reprodutibilidade e validade. Em conformidade com isso, o método deve estar suficientemente detalhado quanto à população/ amostra, às variáveis preditivas e ao desfecho, bem como à análise estatística dos dados ${ }^{4,6}$.

Quanto aos resultados obtidos, a partir do problema de pesquisa, o investigador deve se questionar em relação à aceitabilidade do mesmo em outros contextos de conhecimento, indagando se eles representam acuradamente a pergunta da pesquisa, e se não foram influenciados por fatores de causalidade, tendenciosidade e variáveis intervenientes, por exemplo ${ }^{4,6}$.

Já no âmbito da consistência e confiabilidade dos resultados, a criticidade deve-se ancorar na relevância do achado em termos clínicos, ou seja, a sua finalidade pragmática.

Finalmente, no tocante à avaliação dos dados, a indagação deve-se alicerçar na tomada de decisão, no quanto as informações contribuirão para o cuidado do paciente, ou seja, no quanto os dados possuem aplicabilidade na prática clínica².

Diante do exposto, no processo de elaboração do projeto de pesquisa em nutrição clínica, o pesquisador deve ter em mente a pergunta norteadora, as hipóteses e objetivos, bem como o planejamento do estudo. É através desse planejamento que o estudo se apresentará como exequível ou quimérico; assim, é indispensável descrever minunciosamente o processo de coleta e apuração dos dados, a apresentação dos resultados de forma inteligível, o procedimento estatístico na análise e interpretação dos resultados e, por fim, as conclusões coadunadas com as hipóteses e objetivos definidos no início da investigação.

Nesse sentido, no planejamento do experimento é necessário informar o tipo do estudo e o procedimento estatístico adotado. Inicialmente deve-se definir a natureza do experimento, verificar questões éticas, definir a população e o 
tamanho da amostra, bem como avaliar se ele é factível e os riscos envolvidos na sua condução.

\section{Natureza do experimento}

No que diz respeito à natureza do experimento, Marchioni ${ }^{5}$ e Bloch \& Coutinho ${ }^{6}$ afirmam que os estudos podem ser classificados em dois tipos: experimentais e observacionais. O primeiro se refere aos estudos de intervenção e tem o objetivo de investigar hipóteses de causalidade, avaliar procedimentos terapêuticos ou intervenções preventivas, ou seja, avaliar a alocação dos grupos de exposição e manipulação do fator de exposição. Nessa tipologia, podem ser classificados como estudos randomizados (ensaios clínicos e estudos cruzados) ou estudos de comunidade. Já os estudos observacionais objetivam descrever a distribuição e variáveis de desfecho, e envolvem estudos de coorte, estudos de caso controle, estudos transversais e estudos ecológicos ${ }^{5,6}$.

Na prática clínica em nutrição, os estudos de intervenção, especialmente os ensaios clínicos, são apreciados em investigações causais devido à asseveração da relação causa/efeito e, também, por permitirem elucidar o tratamento mais apropriado para o sujeito. Contudo, tais estudos apresentam limitações quanto a questões éticas, financeiras e a fatores de confundimento. Os fatores de confusão decorrem do fato de a escolha dos sujeitos ser aleatória e o pesquisador não poder nem privá-los de tratamento, nem submetê-los a procedimentos de risco ${ }^{4}$

Com o propósito de controlar os fatores de confusão, a randomização tem sido comumente utilizada para alocação aleatória dos pacientes em grupos de intervenção e controle, objetivando identificar a relação causa/efeito de uma exposição ${ }^{4}$. E, para minimizar vieses relacionados a mudanças de comportamento durante a exposição, bem como inferências equivocadas sobre os resultados, utilizam-se algumas técnicas de mascaramento, a saber: mascaramento a cegas (o paciente desconhece o grupo a que pertence), mascaramento duplo-cego (paciente e investigador desconhecem o grupo a que pertence) ${ }^{4,5} \mathrm{e}$ mascaramento triplo-cego (tanto o profissional que interpreta quanto os demais desconhecem o grupo a que o paciente pertence $)^{7}$.

Os ensaios clínicos podem apresentar delineamentos diversos, como ensaio crossover ou sequencial, ensaio em paralelo, desenho fatorial e ensaio explanatório ${ }^{7}$.

\section{Cálculo do tamanho da amostra}

Dentre as inúmeras dificuldades encontradas pelo pesquisador ao realizar um estudo clínico, destaca-se o cálculo do tamanho amostral de modo que se obtenham resultados que reflitam, de forma clara e precisa, os objetivos da pesquisa ${ }^{8}$.

Como na maior parte dos estudos não há possibilidade de avaliar todos os elementos (pacientes ou indivíduos) de determinada população, principalmente devido ao tempo e custo, o cálculo amostral é de fundamental importância e deve ser realizado durante o planejamento do trabalho, independentemente do tipo de estudo (clínico, experimental, epidemiológico). A amostra deve ser grande o suficiente para atender aos objetivos do estudo, bem como deve ser representativa da população a ser avaliada, de modo que um resultado considerado importante clinicamente apresente significância estatística ${ }^{9,10}$.

O tamanho amostral irá depender de diversos fatores, como: o objetivo do estudo, o tipo da variável de interesse (quantitativa ou qualitativa), as características da população, o poder do teste, o nível de significância adotado e as possíveis perdas durante a realização do estudo. Quando há mais de um desfecho de interesse, o tamanho da amostra deve ser calculado para cada um deles, adotando-se o maior valor ${ }^{11,12}$.

Existem diversos métodos de amostragem (processo de escolha dos indivíduos que farão parte da amostra). Os mais utilizados são os métodos aleatórios ou probabilísticos, que permitem 
que todos os indivíduos da população estudada tenham a mesma chance de fazer parte da amostra; assim, devem ser priorizados, para garantir a qualidade da amostra. A amostragem aleatória pode ser simples, sistemática, estratificada ou por conglomerado?.

O processo de amostragem não garante que uma amostra seja perfeitamente representativa da população avaliada; assim, implica a aceitação de uma margem de erro, denominado erro amostral ou aleatório. Esse tipo de erro é definido como a distância máxima permitida entre o valor de um parâmetro da população e o valor amostral do mesmo parâmetro. Existe uma relação inversa entre o erro e o tamanho da amostra, ou seja, quanto maior a amostra, menor será o erro amostral13.

O outro tipo de erro, denominado não amostral ou sistemático, pode ser evitado ou reduzido durante o planejamento e a execução da pesquisa. As principais causas desse tipo de erro nos estudos são as falhas na coleta, digitação e análise dos dados, bem como as falhas nos instrumentos de coleta ${ }^{13}$.

Na fase de análise estatística dos dados, o pesquisador está sujeito a dois tipos de erros inerentes a esse processo: o erro tipo I ou alfa $(\alpha)$, e o erro tipo II ou beta ( $\beta$ ). $O \alpha$, também denominado como nível de significância, é a probabilidade de considerar como significante uma diferença entre grupos, quando na verdade essa não existe (falso positivo). A maioria dos estudos considera valores de $\alpha$ menores que 0,05 ou $5 \%$; assim, a probabilidade máxima de erro aceita para se rejeitar a hipótese de nulidade (igualdade) é de $5 \%{ }^{9,14}$.

O $\beta$ é a probabilidade de não se encontrar uma diferença significante entre os grupos do estudo, quando ela de fato existe, classificando-os erroneamente como iguais (falso negativo). Outro termo comumente utilizado é o "poder do teste", definido como (1- $\beta$ ), que expressa a probabilidade de detectar um resultado verdadeiro. Convencionalmente, o poder do teste nos estudos tem sido estabelecido como $80 \%$ ou $90 \%(0,8$ ou 0,9). Assim, para o poder ser no mínimo igual a 0,8 , a probabilidade de ocorrer o erro tipo II não deve ser maior do que $0,2(20 \%)^{10,14,15}$.

Muitos estudos clínicos têm como objetivo a comparação entre duas proporções - por exemplo, avaliar se a proporção de cura de determinada doença é diferente ou não entre os pacientes expostos a alguma intervenção (variáveis dicotômicas). Essa abordagem é também utilizada em outros tipos de estudo, sempre que o objetivo for a comparação entre duas proporções. É também frequente a realização de estudos clínicos cujo interesse é a comparação de duas médias, como exemplo, avaliar se existe diferença entre dois tratamentos alternativos para o controle de uma variável numérica, como a glicemia ${ }^{9,16}$.

\section{Comparação entre duas proporções}

Para determinação do tamanho da amostra quando o objetivo é a comparação entre duas proporções, é necessário que se defina o $\alpha$ e o $\beta$ e que se conheça a estimativa das duas proporções

$n=\frac{\left(z_{1-\frac{\alpha}{2}} \sqrt{2 \overline{p(1-\bar{p})}}+z_{1-\beta} \sqrt{p_{1}^{\left(1-p_{1}\right)}+p_{2}^{\left(1-p_{2}\right)}}\right)^{2}}{\left(\bar{p}_{1}-\bar{p}_{2}\right)^{2}}$

onde: $n$ : tamanho da amostra; $\mathrm{Z}=$ valor da distribuição normal para determinado nível de confiança (Tabela 1); $\alpha$ : erro tipo I; $\beta$ : erro tipo II: $Z$ : valor da distribuição normal para determinado nível de confiança (Tabela 1); $p_{1}$ : proporção do desfecho no grupo $1 ; p_{2}$ : proporção do desfecho no grupo $2 ; p:\left(p_{1}+p_{2}\right) / 2$.

\section{Comparação entre duas médias}

Para determinação do tamanho da amostra quando se deseja comparar duas médias, é necessário que se defina o $\alpha$ e o $\beta$, e que se conheça o desvio-padrão da população que será investigada e a diferença clinicamente relevante entre as duas médias: 
Tabela 1. Grau de confiança, erro tipo I $(\alpha)$, erro tipo $2(\beta)$, poder do estudo (1- $\beta$ ) e valores da distribuição normal $(Z)$ para $Z_{\alpha / 2}$ e $Z_{\beta}$.

\begin{tabular}{lccccc}
\hline Grau de confiança & $\alpha$ & $Z_{\alpha / 2}$ & $\beta$ & $1-\beta$ & $Z_{\beta}$ \\
\hline $90 \%$ & 0,10 & 1,65 & 0,2 & 0,8 & 0,84 \\
$95 \%$ & 0,05 & 1,96 & 0,1 & 0,9 & 1,28 \\
$99 \%$ & 0,01 & 2,58 & 0,05 & 0,95 & 1,65 \\
\hline
\end{tabular}

Fonte: Martínez-González et al. ${ }^{18}$.

$$
\frac{n=2 S^{2}\left(t_{\frac{a}{2} ; g l=2 n-1}+t_{\beta ; g l=2 n-1}\right)^{2}}{d^{2}}
$$

onde: $\mathrm{n}$ : tamanho da amostra; a: erro tipo I; b: erro tipo II; $S$ : desvio-padrão; $d$ : diferença a detectar; $t_{1}$ : média $1 ; t_{2}$ : média 2.

Em algumas situações, é necessário que se façam ajustes no cálculo do tamanho da amostra, a depender do tamanho da população, do desenho amostral e das perdas de seguimento.

\section{Ajuste para tamanho da população}

O tamanho amostral calculado deve ser aplicado a uma população infinita. Caso a população estudada possua menos que 2 mil elementos, é necessário que se faça ajuste no tamanho da amostra ${ }^{10}$.

$$
\begin{aligned}
& \mathrm{n}_{\text {ajustado }}=\mathrm{n} / 1+(\mathrm{n} / \mathrm{N}) \\
& \mathrm{n}=\text { tamanho amostral calculado } \\
& \mathrm{N}=\text { tamanho da população }
\end{aligned}
$$

\section{Ajuste para efeito do desenho amostral}

Quando o tamanho da amostra é calculado para amostragem aleatória simples e caso a amostra seja selecionada por outro método, como amostragem por conglomerado, deve-se corrigir o tamanho amostral calculado, que é chamado de correção do efeito do desenho (deff) 9 .

$\mathrm{n}_{\text {deff }}=\mathrm{n} \times$ deff

$\mathrm{n}=$ tamanho amostral calculado

deff $=$ efeito do desenho*

*Sugere-se, para amostragem por conglomerado, deff $=1,5$

\section{Ajuste para possíveis perdas de seguimento}

Durante o estudo, as perdas de elementos superiores à estimada podem comprometer a validade interna da pesquisa. A perda de elementos é comum principalmente em pesquisas de delineamento longitudinal. Nesses casos, o tamanho da amostra deve ser corrigido pela proporção estimada da perda.

$$
\begin{aligned}
& \mathrm{n}_{\text {ajustado }}=\mathrm{n} / 1 \text { - proporção estimada de perda } \\
& \mathrm{n}=\text { tamanho amostral calculado }
\end{aligned}
$$

\section{Análise dos dados}

Realizada a coleta e digitação das informações em um banco de dados, a etapa seguinte consiste na análise exploratória ou descritiva. A análise descritiva permite ao investigador conhecer melhor os seus dados, organizá-los e sintetizá-los, além de indicar possíveis modelos a serem aplicados na etapa posterior de análise, denominada inferência estatística. Permite também identificar os possíveis outliers (casos atípicos) e avaliar a ausência de dados (missing) ou sua distribuição na curva de Gauss (normalidade) ${ }^{17}$.

Os dados podem ser apresentados em tabelas, gráficos ou mapas, dependendo do objetivo do estudo. A descrição dos resultados nessa etapa pode ser feita por distribuição de frequências (variáveis qualitativas), estimação de medidas de tendência central (média e mediana) e medidas de dispersão (desvio-padrão, percentis, valores mínimo e máximo) ${ }^{17}$.

A estatística inferencial, que procede à análise descritiva, permite realizar inferências 
Quadro 2. Exemplos de testes estatísticos, de acordo com o objetivo do estudo e a distribuição das variáveis na curva de Gauss.

\begin{tabular}{|c|c|c|}
\hline Objetivo & Teste paramétrico & Teste não paramétrico \\
\hline Verificar a relação entre duas variáveis numéricas & Correlação de Pearson & Correlação de Spearman \\
\hline Comparar dois grupos independentes & Teste $t$ de Student & Mann-Whitney \\
\hline Comparar três ou mais grupos independentes & Análise de variância & Kruskal-Wallis \\
\hline Comparar dois grupos dependentes & Teste $t$ pareado & Wilcoxon \\
\hline Comparar três ou mais grupos dependentes & Análise de variância de medidas repetidas & Friedman \\
\hline Verificar a associação entre duas variáveis categóricas & - & $\begin{array}{l}\text { Qui-quadrado de Pearson ou Exa- } \\
\text { to de Fisher }\end{array}$ \\
\hline Verificar a magnitude da associação (causalidade) & \multicolumn{2}{|c|}{$\begin{array}{c}\text { Risco relativo } \\
\text { Razão de prevalência } \\
\text { Odds Ratio }\end{array}$} \\
\hline
\end{tabular}

(conclusões), a partir de dados de uma amostra, para toda a população. Diferentes testes estatísticos podem ser aplicados nessa etapa da análise, sendo a escolha dependente dos objetivos do estudo e do tipo de dados ${ }^{19}$.

No Quadro 2 estão exemplificados alguns testes estatísticos, de acordo com o objetivo e a distribuição das variáveis na curva de Gauss (testes paramétricos ou não paramétricos) $)^{17,19}$.

\section{CONSIDERAÇÕ ES FINAIS}

A prática clínica em nutrição requer do profissional conhecimento amplo sobre os problemas clínicos, bem como a definição de qual o procedimento adequado para tratamento do paciente. A prática baseada em evidências tem subsidiado a tomada de decisões de diversas áreas da saúde, por avaliar, organizar e sistematizar os resultados para a tomada de decisão. Além disso, possibilita verificar se a magnitude dos resultados na prática clínica é significativa, embora as análises estatísticas a apresentem.

Assim, é imprescindível que os estudos sejam válidos, relevantes, confiáveis e apresentem evidências para o cuidado do paciente. A fim de minimizar vieses e variáveis de confusão em estudos clínicos, torna-se indispensável o cálculo amostral, pois ele irá determinar se o resultado tem importância clínica e significância estatística.
Diante do exposto, são muitos os critérios a serem avaliados antes da realização de um estudo clínico, iniciando-se pela denição do tema, objetivos e metodologia (cálculo amostral, método de amostragem, análise estatística), além da vericação da consistência dos resultados e, ao final, sua adequada interpretação.

\section{COLABORADORES}

DAA PEREIRA e SA VIEIRA projetaram o artigo, realizaram levantamento e interpretação da literatura e elaboração do manuscrito. AS FOGAL participou da elaboração do artigo e revisão do manuscrito. AQ RIBEIRO e SCC FRANCESCHINI orientaram e supervisionaram o artigo e fizeram correções técnicas.

\section{REFERÊ N CIAS}

1. Santos CMC, Pimenta CAM, Nobre MRC. A estratégia pico para a construção da pergunta de pesquisa e busca de evidências. Rev Latino-Am Enferm. 2007; 3(15):1-4. doi: 10.1590/S0104-116 92007000300023

2. Cruz DALM, Pimenta CAM. Prática baseada em evidências, aplicada ao raciocínio diagnóstico. Rev Latino-Am Enferm. 2005; 13(3):415-22. doi: 10.1590/S0104-11692005000300017

3. Stetler $C B$, Morsi D, Rucki S, Broughton S, Corrigan B, Fitzgerard J, et al. Utilization-focused integrative reviews in a nursing service. Applied Nursing Res. 1998; 11(4):195-206. doi: 10.1016/50897-1897 (98)80329-7 
4. Werneck GL, Almeida LM. Validade em estudos epidemiológicos. In: Medronho AR, Carvalho DM, Bloch KV, Luiz RR, Werneck GL. Epidemiologia. São Paulo: Atheneu; 2011.

5. Marchioni DML. Estudos epidemiológicos em exposições nutricionais. In: Fisberg RM, Slater B, Marchioni DML, Martini LA, Organizador. Inquéritos alimentares: métodos e bases científicas. Barueri: Manole; 2011.

6. Bloch KV, Coutinho ESF. Fundamentos da pesquisa epidemiológica. In: Medronho AR, Carvalho DM, Bloch KV, Luiz RR, Werneck GL. Epidemiologia. São Paulo: Atheneu; 2011. p.173-9.

7. Escosteguy. Estudos de intervenção. In: Medronho AR, Carvalho DM, Bloch KV, Luiz RR, Werneck GL. Epidemiologia. São Paulo: Atheneu; 2011. p.251-63.

8. Marotti J, Galhardo APM, Furuyama RJ, Pigozzo MN, Campos TN, Lagana DC. Amostragem em pesquisa clínica: tamanho da amostra. Rev Odontol Univ Cid. São Paulo. 2008; 20(2):186-94.

9. Luiz RR, Magnanini MMF. O tamanho da amostra em investigações epidemiológicas. In: Medronho AR, Carvalho DM, Bloch KV, Luiz RR, Werneck GL. Epidemiologia. São Paulo: Atheneu; 2011. p.415-27.

10. Normando D, Almeida MAO, Quintão CCA. Análise do emprego do cálculo amostral e do erro do método em pesquisas científicas publicadas na literatura ortodôntica nacional e internacional. Dental Press J Orthod. 2011; 16(6):1-9. doi: 10.15 90/S2176-94512011000600006

11. Breslow NE. Statistics in epidemiology: The case-control study. J Am Statist Assoc. 1996; 91(433):14-28. doi: $10.1080 / 01621459.1996 .10476660$
12. Hulley SB, Browner WS, Cummings SR, Grady D, Newman TB. Delineando a pesquisa clinica: uma abordagem epidemiológica. Porto Alegre: Artmed; 2008.

13. Torres TZG, Magnanini MMF, Luiz RR. Amostragem. In: Medronho AR, Carvalho DM, Bloch KV, Luiz RR, Werneck GL. Epidemiologia. São Paulo: Atheneu; 2011. p.403-14.

14. MacFarlane TV. Sample size determination for research projects. J Orthod. 2003; 30(2):99-100. doi: 10.1093/ortho/30.2.99

15. Schulz KF, Grimes DA. Sample size calculations in randomised trials: Mandatory and mystical. Lancet. 2005; 365(9467):1348-53. doi: 10.1016/S0 140-6736(05)61034-3

16. Agranonik M, Hirakata VN. Cálculo de tamanho de amostras: proporções. Rev HCPA. 2011; 33(1):382-8.

17. Pinheiro RS, Torres TZG. Análise exploratória de dados. In: Medronho AR, Carvalho DM, Bloch KV, Luiz RR, Werneck GL. Epidemiologia. São Paulo: Atheneu; 2011. p.323-42.

18. Martínez-González MA, Sánchez-Villegas A, Fajardo JF. Bioestadística Amigable. $2^{\text {a }}$ ed. Madri: Diaz de Santos; 2009.

19. Callegari-Jackes, SM. Testes não-paramétricos. In: Callegari-Jackes SM. Bioestatística: princípios e aplicações. Porto Alegre: Artmed; 2003. p.165-84.

Recebido em: 16/5/2014 Versão final em: 4/9/2014 Aprovado em: 6/11/2014 